\title{
Effect of Surfactant on Homogeneity of Partially Degummed Silk Fiber
}

\author{
Da Eun Chung and In Chul Um* \\ Department of Bio-fibers and Materials Science, Kyungpook National University, Daegu 702-701, Republic of Korea
}

\section{Abstract}

Silk has always been one of the most favored textile materials. Fully degummed silk fiber (i.e., silk fibers without sericin) shows better luster than raw silk fiber (with sericin); it is also softer. On the other hand, raw silk fiber feels cooler because of the presence of sericin, making it useful as a textile for the summer season. Recently, partially degummed silk has attracted researchers' attention because it provides better luster, feel, and dyeing properties. However, the partial degumming of silk is very difficult because it results in inhomogeneously degummed fiber. In the present study, silk yarns were degummed with surfactant aqueous solutions and the effects of each surfactant on the degumming ratio, crystallinity, and homogeneity of the degummed silk yarn were examined. The degumming ratio and crystallinity index of silk yarn varied depending on the type of surfactant. On the whole, anionic surfactants resulted in higher degumming ratios and better homogeneity than nonionic surfactants.

Received : 25 Feb 2014

Accepted : 25 Mar 2014

Keywords:

Silk,

Degumming,

Homogeneity,

Surfactant

\section{Introduction}

Silk is a highly sought-after textile material because of its excellent luster and because it feels good to the skin. Silk is a naturally occurring composite fiber consisting of two fibroin strands with a sericin covering. Removal of the sericin by degumming decreases the diameter of the silk fiber, resulting in finer fibers and thus better texture. In addition, the fibroin exhibits a more regular cross-sectional (i.e., triangular) shape after the degumming process. This significantly improves the luster of the silk fibroin. Thus, owing to the improvement of luster and texture of silk fiber by degumming, sericin is usually removed from silk fiber in textile manufacturing.

Recently, however, raw silk fiber containing sericin has attracted consumers as a textile material suitable for summers because of cooling effect of the raw silk fiber; researchers have also realized that the two different types of silk fiber, raw silk with sericin and degummed silk fiber without sericin, have different properties when used as textiles. Therefore, researchers have tried to produce partially degummed silk fiber with properties intermediate between those of raw silk and fully degummed silk.

However, the preparation of homogeneously partially degummed silk is difficult. Park (2010) reported the difficulties involved in evenly dissolving and removing sericin from the surface of raw silk fiber. Ko et al. (2013) showed the heterogeneous sericin removal of partially degummed silk by scanning electron microscopy (SEM). Lee et al. (2003) conducted the partial degumming of silk and reported that the

\section{*Corresponding author.}

\section{In Chul Um}

Department of Bio-fibers and materials Science (Functional Fiber \& Material Lab), College of Agriculture and Life Science, Kyungpook National University, 1370, Sangyeok-dong, Buk-gu, Daegu 702-701, South Korea

Tel: +82-53-950-7757 / FAX: +82-53-950-6744

E-mail: icum@knu.ac.kr 
abrasion resistance and drape coefficient of silk deteriorated with increasing degumming ratio. Kim et al. (2013) used Fourier transform infrared (FTIR) spectroscopy to examine the homogeneity of degumming and investigated the effect of degumming conditions on the homogeneity of partially degummed silk fiber as a preliminary study. They reported that the addition of a surfactant (sorbitan monostearate) improved the homogeneity of silk degumming.

As a follow-up to our previous study, we used nonionic and anionic surfactants and examined their effects on the homogeneity of partially degummed silk fibroin using FTIR spectroscopy.

\section{Materials and Methods}

\section{Degumming}

In the present study, silk yarns were used as silk sample and they was provided by Sanju Myungju Company. A single silk

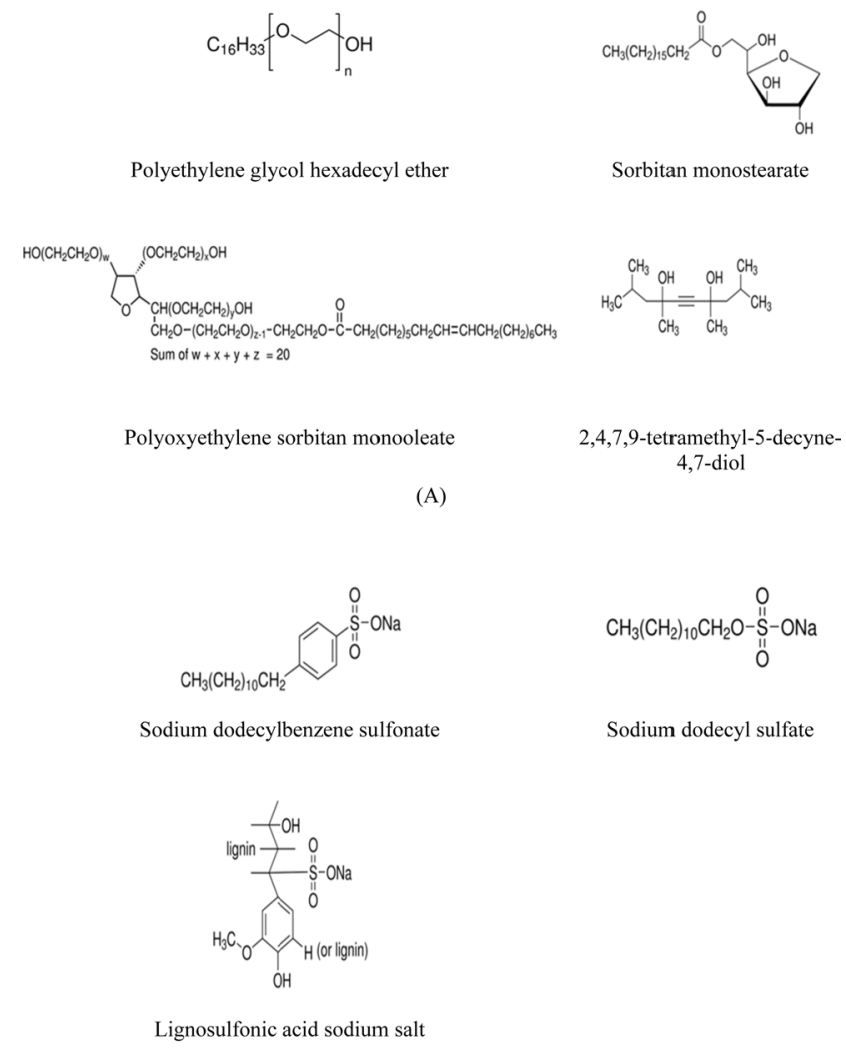

(B)

Fig. 1. Chemical structures of (A) nonionic surfactants and (B) anionic surfactants . yarn consists of five silk fibers with 21 denier. The degumming method used has been reported elsewhere (Kim et al., 2013). Briefly, four nonionic surfactants (polyethylene glycol hexadecyl ether, polyoxyethylene sorbitan monooleate, sorbitan monostearate, and 2,4,7,9-tetramethyl-5-decyne-4,7-diol) and three anionic surfactants (sodium dodecylbenzene sulfonate, lignosulfonic acid sodium salt, and sodium dodecyl sulfate) were used. The chemical structures of the surfactants are shown in Fig. 1. Silk yarns were degummed with an $8 \%$ [o.w.f. (on the weight of fiber)] surfactant aqueous solution at $100^{\circ} \mathrm{C}$ for $1 \mathrm{~h}$. The liquor ratio was 1:200. After the degumming was completed, the degummed silk yarns were rinsed thoroughly in warm distilled water to remove extra surface-associated sericin and then dried. The distilled water was obtained using a water purification system (RO50, Hana Science, South Korea) with a reverse osmosis membrane. The dry mass of the silk yarns was measured using a moisture analyzer (XM60, Precisa, Switzerland). The degumming ratio was calculated using the following equation:

Degumming ratio $(\%)=[1-($ dry mass of degummed yarns $/$ dry mass of raw silk yarns) $] \times 100$.

\section{Measurement}

The FTIR measurements and method of determining the crystallinity index have been reported elsewhere (Kim et al., 2013). Briefly, FTIR-ATR spectra were obtained on an FTIR spectrometer (Nicolet 380, Thermo Fisher Scientific, USA). The crystallinity index was calculated as the intensity ratio of the 1260 and $1235 \mathrm{~cm}^{-1}$ peaks in the FTIR spectrum using the following equation:

Crystallinity index $(\%)=\frac{A_{1260 \mathrm{~cm}^{-1}}}{A_{1235 \mathrm{~cm}^{-1}}} \times 100$

A $1235 \mathrm{~cm}^{-1}$ : Absorbance at $1235 \mathrm{~cm}^{-1}$

A $1260 \mathrm{~cm}^{-1}:$ Absorbance at $1260 \mathrm{~cm}^{-1}$

To determine the average and variation of the crystallinity index, FTIR measurements were performed at 30 different points along each silk yarn sample. The degumming inhomogeneity of the silk yarn was calculated using the following equation:

Degumming inhomogeneity $(\%)=($ standard deviation of the crystallinity index $) /($ average of the crystallinity index $) \times 100$ 


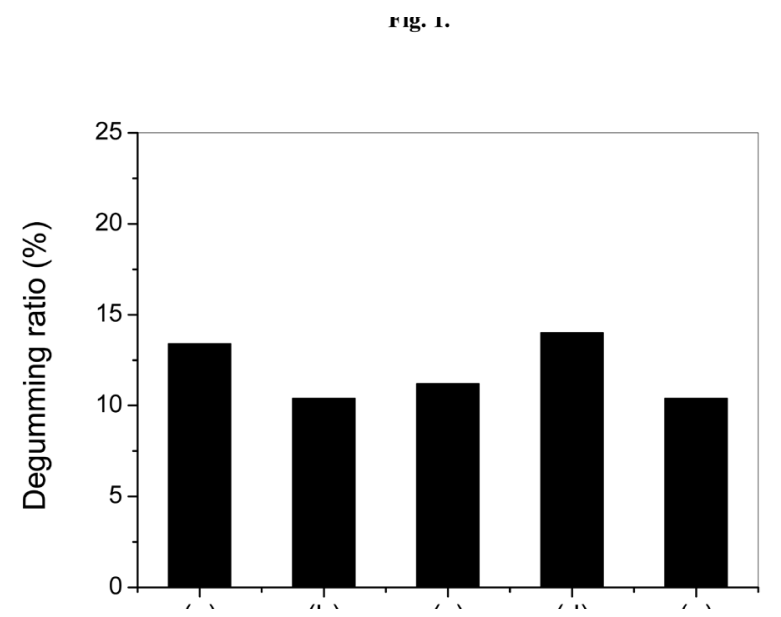

Fig. 2. Degumming ratio of silk yarn degummed with $8 \%$ (o.w.f.) nonionic surfactant aqueous solutions: (a) no surfactant, (b) polyethylene glycol hexadecyl ether, (c) polyoxyethylene sorbitan monooleate, (d) sorbitan monostearate, and (e) 2,4,7,9-tetramethyl5-decyne-4,7-diol.

\section{Results and Discussion}

\section{Effect of surfactant on degumming ratio and crystallinity of silk yarn}

Fig. 2 shows the degumming ratio of silk yarn degummed with nonionic surfactants. The degumming ratio of the silk yarn ranged from $10 \%$ to $15 \%$. Except for sorbitan monostearate (14\% degumming ratio), the nonionic surfactants resulted in lower degumming ratios (10-12\%) than the aqueous solution (i.e., without surfactant, 13.4\%). Fig. 3 shows the degumming ratio of silk yarn degummed with anionic surfactants. As can be seen in the figure, anionic surfactants showed higher degumming ratios (15-18\%) than the aqueous solution (13.4\%). These results indicate that the use of surfactants can affect the degumming ratio of silk. On the whole, anionic surfactants show higher degumming ratios than nonionic surfactants. It is assumed that this is related to hydrophilicity of anionic surfactants. Silk sericin is composed of various amino acids and has polar groups. Therefore, hydrophilic anionic surfactants can dissolve sericin more easily in the degumming bath than nonionic surfactant, resulting in higher degumming ratios.

To examine the effect of the nonionic surfactants on the molecular conformation and crystallinity of degummed silk yarn, FTIR measurements were performed on the degummed silk yarns (Fig. 4). Regardless of the nonionic surfactant type, all the

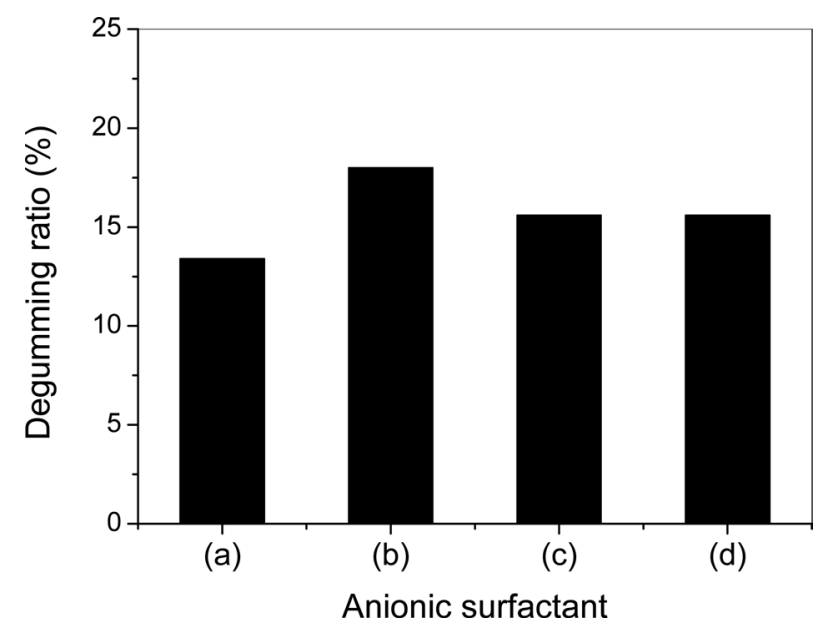

Fig. 3. Degumming ratio of silk yarn degummed with $8 \%$ (o.w.f.) anionic surfactant aqueous solutions: (a) no surfactant, (b) sodium dodecylbenzene sulfonate, (c) lignosulfonic acid sodium salt, and (d) sodium dodecyl sulfate.

degummed silk yarns showed similar FTIR spectra [Fig. 4(A)]. That is, all silk yarns showed strong IR absorption peaks at 1620 and $1512 \mathrm{~cm}^{-1}$, which is attributed to $\beta$-sheet conformation (Lee et al., 2013; Um et al., 2001; Um et al., 2003). Also, a shoulder absorption peak at $1260 \mathrm{~cm}^{-1}$ due to $\beta$-sheet conformation appeared; the intensity of this peak slightly differs depending on the surfactant type. To examine the molecular conformation more quantitatively, the crystallinity index was calculated from the absorption peaks at 1260 and $1235 \mathrm{~cm}^{-1}$ (Fig. 4(B)). The crystallinity index of the degummed silk yarn showed similar values except for that degummed using polyethylene glycol hexadecyl ether. When the same degumming agent was used, the crystallinity index of silk increased with increasing degumming ratio (Kim et al., 2013). However, when the degumming ratio (Fig. 2) and crystallinity index [Fig. 4(B)] of the silk yarns degummed with nonionic surfactants are compared, they do not show good correlation. This indicates that it is difficult to directly correlate the degumming ratio and crystallinity index when the degumming agent is different.

Fig. 5 shows the FTIR spectra and crystallinity index of silk yarn degummed with anionic surfactants. The FTIR spectra were similar to those of the nonionic surfactants, and there was no significant difference among the nonionic surfactants [Fig. 5(A)]. However, the crystallinity index provided more detailed information about the microstructure of the silk. Sodium dodecylbenzene sulfonate showed the highest crystallinity index among the surfactants. These FTIR 


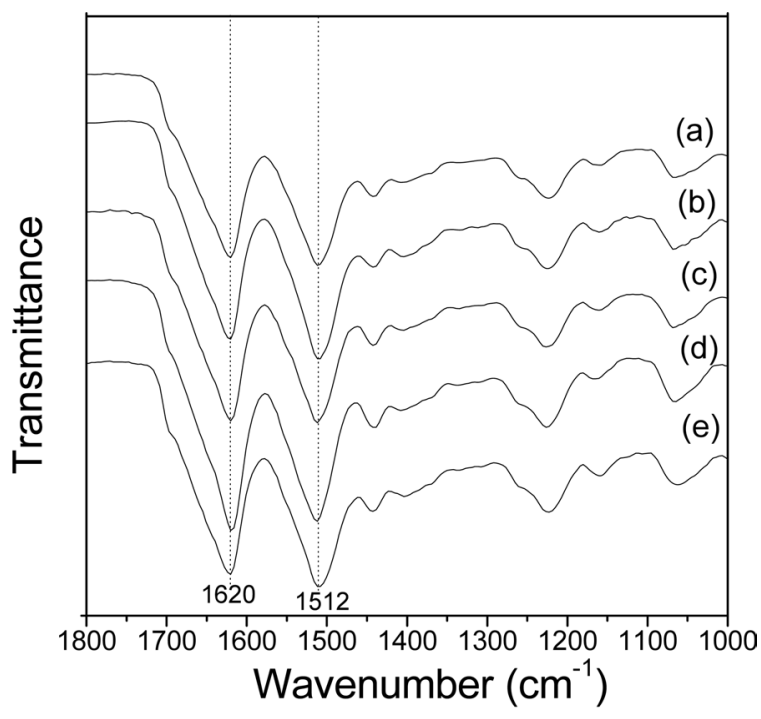

(A)

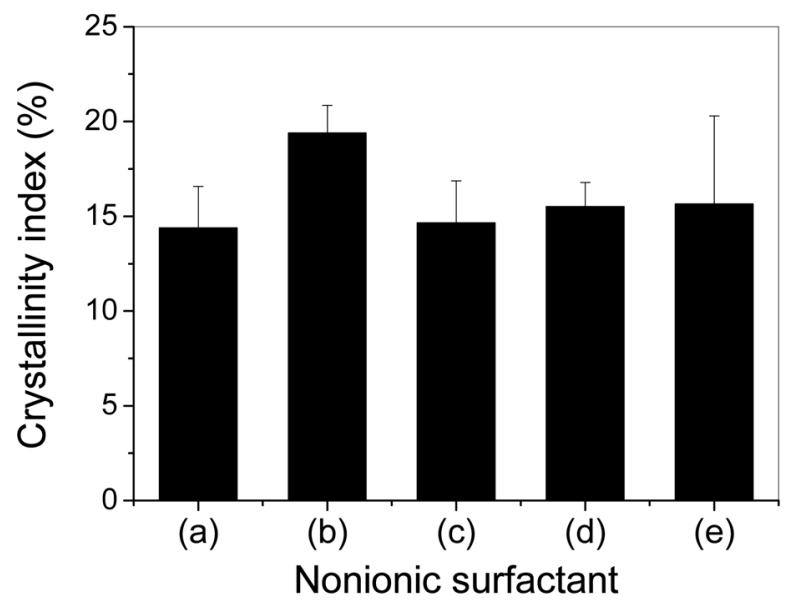

(B)

Fig. 4. (A) FTIR spectra and (B) crystallinity index of silk yarn degummed with $8 \%$ (o.w.f.) nonionic surfactant aqueous solutions: (a) no surfactant, (b) polyethylene glycol hexadecyl ether, (c) polyoxyethylene sorbitan monooleate, (d) sorbitan monostearate, and (e) 2,4,7,9-tetramethyl-5-decyne-4,7-diol.

results indicate that the use of anionic surfactants led to changes in the crystallinity index of silk yarn and that the crystallinity index varied depending on the type of surfactant.

\section{Effect of surfactant on homogeneity of degumming}

Kim et al. (2013) evaluated the homogeneity of degumming using FTIR. They examined the degumming homogeneity by the standard deviation of FTIR crystallinity index. In this study, the degumming inhomogeneity was calculated by an equation

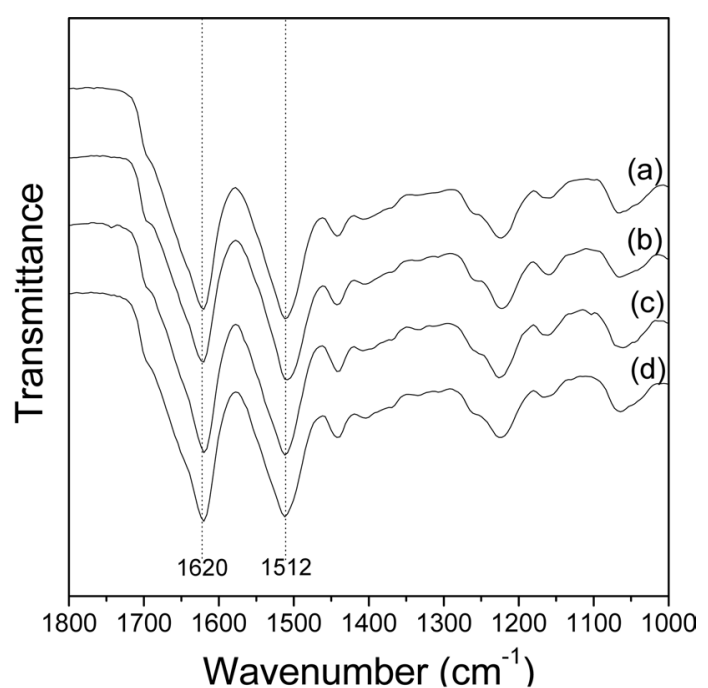

(A)

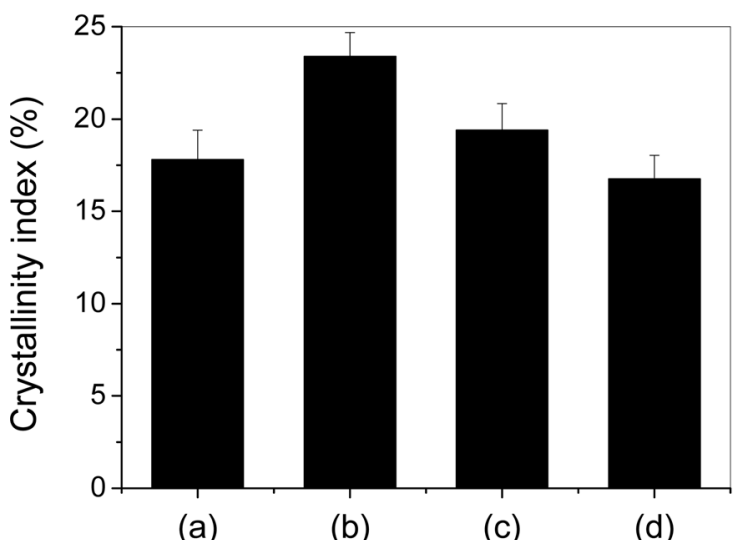

Anionic surfactant

(B)

Fig. 5. (A) FTIR spectra and (B) crystallinity index of silk yarn degummed with $8 \%$ (o.w.f.) anionic surfactant aqueous solution: (a) no surfactant, (b) sodium dodecylbenzene sulfonate, (c) lignosulfonic acid sodium salt, and (d) sodium dodecyl sulfate.

[standard deviation of the crystallinity index)/(average of the crystallinity index) $\mathrm{x}$ 100] as introduced in experimental part to examine the homogeneity of degumming quantitatively. By this equation, the homogeneity of degumming is improved by decreasing the degumming inhomogeneity.

Fig. 6 shows the degumming inhomogeneity of silk yarn degummed with nonionic surfactants. Silk yarn degummed with 2,4,7,9-tetramethyl-5-decyne-4,7-diol showed a higher degumming inhomogeneity than that degummed with an aqueous solution, indicating that degumming homogeneity 


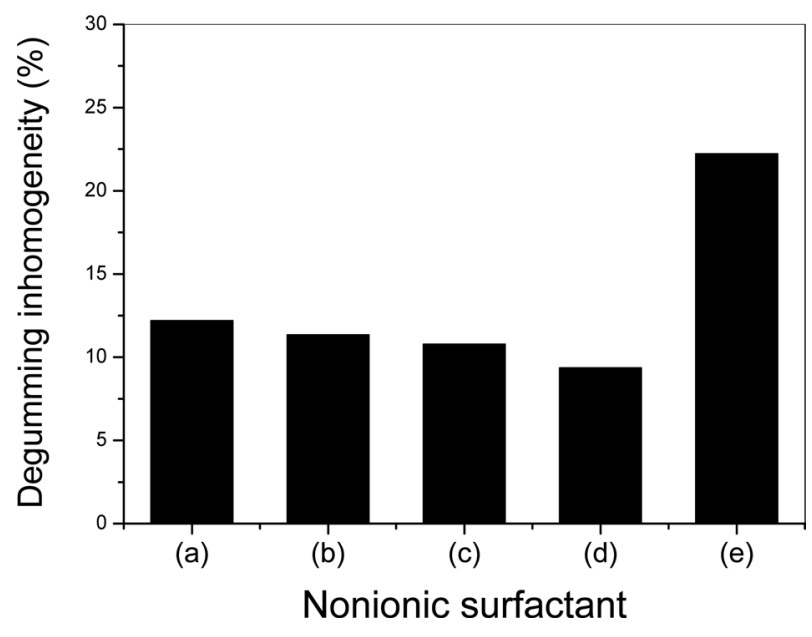

Fig. 6. Degumming inhomogeneity of silk yarn degummed with $8 \%$ (o.w.f.) nonionic surfactant aqueous solutions: (a) no surfactant, (b) polyethylene glycol hexadecyl ether, (c) polyoxyethylene sorbitan monooleate, (d) sorbitan monostearate, and (e) 2,4,7,9-tetramethyl5-decyne-4,7-diol.

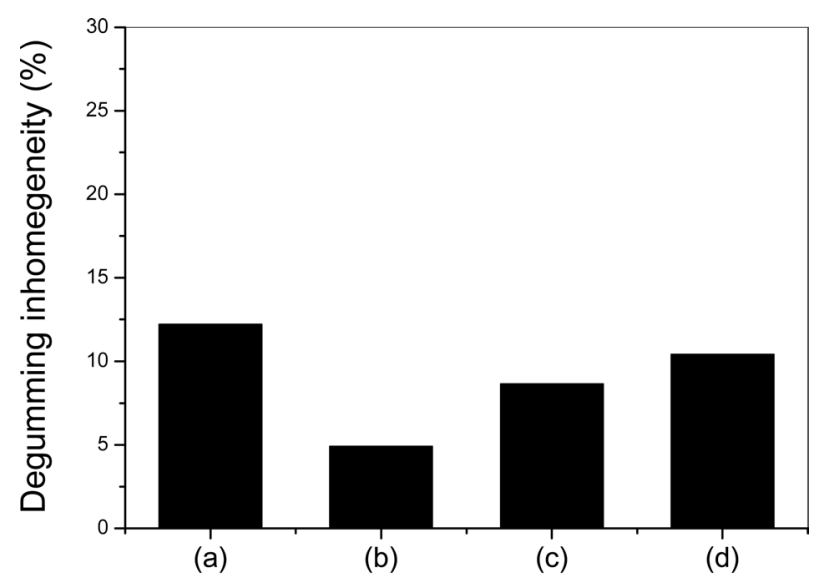

Anionic surfactant

Fig. 7. Degumming inhomogeneity of silk yarn degummed with $8 \%$ (o.w.f.) anionic surfactant aqueous solutions: (a) no surfactant, (b) sodium dodecylbenzene sulfonate, (c) lignosulfonic acid sodium salt, and (d) sodium dodecyl sulfate.

deteriorated by using 2,4,7,9-tetramethyl-5-decyne-4,7-diol. Other nonionic surfactants (polyethylene glycol hexadecyl ether, polyoxyethylene sorbitan monooleate, and sorbitan monostearate) showed a slightly lower degumming inhomogeneity than the aqueous solution, implying that these surfactants can be utilized to slightly improve the degumming homogeneity.
When the anionic surfactants were added to the water degumming bath, the homogeneity of degumming was more enhanced. As can be seen in Fig. 7, all the anionic surfactants showed smaller degumming inhomogeneity, indicating that the degumming was conducted more homogeneously with these surfactants than with the aqueous solution. In particular, sodium dodecylbenzene sulfonate showed a significantly lower degumming inhomogeneity than others, indicating that this surfactant is the most effective surfactant for improving the homogeneity of silk degumming among all the surfactants used in the present study.

On the whole, anionic surfactants showed better performance in terms of degumming homogeneity than nonionic surfactants. Ionic surfactant has a high hydrophilicity. Thus, it seems that the ionic surfactant can penetrate the raw silk yarns faster and more evenly than water only resulting in more homogenous removal of sericin from silk yarns.

In the present study, various surfactants were evaluated to improve the homogeneity of silk degumming, and it was found that the surfactants enhanced the degumming homogeneity. It was also found that the performance of the surfactant depends on the type of surfactant. Therefore, it is necessary to carefully choose the surfactants for silk degumming.

\section{Acknowledgement}

This study was supported by the Basic Science Research Program (2012042016) and the "Leaders in INdustry-university Cooperation" Project through the National Research Foundation of Korea (NRF) funded by the Ministry of Education, Science and Technology (MEST).

\section{References}

Kim HJ, Chung DE, Um IC (2013) Homogeneity of degumming of silk with different degumming conditions. Int J Indust Entomol 26, 54-60. Ko JS, Yoon K, Ki CS, Kim HJ, Bae DG, Lee KH, Park YH, Um IC (2013) Effect of degumming condition on the solution properties and electrospinnablity of regenerated silk solution. Int J Biol Macromol 55, 161-168.

Lee EM, Lee HJ, Yoo HJ (2003) The physical properties and dyeability 
Da Eun Chung and In Chul Um
Effect of surfactant on homogeneous degumming

of degummed and sericin fixed silk fabrics. J Kor Soc Cloth Text 27, 517-523.

Lee JH, Bae CH, Park BD, Um IC (2013) Preparation of cellulose nanofibril/regenerated silk fibroin composite fibers. Int J Indust Entomol 26, 81-88.

Park GY (2010) Half-degumming behaviors of raw silk yarns degummed with soap and alkalis. Text Color Finish 22, 349-355.
Um IC, Kweon H, Park YH, Hudson S (2001) Structural characteristics and properties of the regenerated silk fibroin prepared from formic acid. Int J Biol Macromol 29, 91-97.

Um IC, Kweon HY, Lee KG, Park YH (2003) The role of formic acid in solution stability and crystallization of silk protein polymer. Int J Biol Macromol 33, 203-213. 